\title{
Do General Circulation Models Underestimate the Natural Variability in the Arctic Climate?
}

\author{
D. S. BAtTisti AND C. M. Bitz \\ Department of Atmospheric Sciences, University of Washington, Seattle, Washington \\ R. E. MORITZ \\ Polar Science Center, Applied Physics Laboratory, University of Washington, Seattle, Washington
}

(Manuscript received 5 February 1996, in final form 11 July 1996)

\section{ABSTRACT}

\begin{abstract}
The authors examine the natural variability of the arctic climate system simulated by two very different models: the Geophysical Fluid Dynamics Laboratory (GFDL) global climate model, and an area-averaged model of the arctic atmosphere-sea ice-upper-ocean system called the polar cap climate model, the PCCM. A 1000-yr integration of the PCCM is performed in which the model is driven by a prescribed, stochastic atmospheric energy flux convergence $(D)$, which has spectral characteristics that are identical to the spectra of the observed $D$. The standard deviation of the yearly mean sea ice thickness from this model is $0.85 \mathrm{~m}$; the mean sea ice thickness is $3.1 \mathrm{~m}$. In contrast, the standard deviation of the yearly averaged sea ice thickness in the GFDL climate model is found to be about $6 \%$ of the climatological mean thickness and only $24 \%$ of that simulated by the PCCM.

A series of experiments is presented to determine the cause of these disparate results. First, after changing the treatment of sea ice and snow albedo in the (standard) PCCM model to be identical thermodynamically to that in the GFDL model, the PCCM is driven with $D$ from the GFDL control integration to demonstrate that the PCCM model produces an arctic climate similar to that of the GFDL model. Integrations of the PCCM are then examined in which the different prescriptions of the sea ice treatment (GFDL vs standard PCCM) and $D$ (GFDL vs observed) are permutated. The results indicate that unarguable improvements in the treatment of sea ice in the GFDL climate model should amplify significantly the natural variability in this model. The authors present calculations that indicate the variability in the sea ice thickness is extremely sensitive to the spectrum of the atmospheric energy flux convergence. Specifically, the differences between the GFDL and observed $D$ at timescales shorter than $3 \mathrm{yr}$ are shown to have a significant impact on the sea ice variability on all timescales. A conservative best estimate for the amplitude of the natural variability in the arctic sea ice volume is presented; this estimate is a significant fraction (about 25\%) of the mean sea ice thickness.

The results suggest that most of the global climate models that have been used to evaluate climate change may also have artificially quiescent natural variability in the Arctic.
\end{abstract}

\section{Introduction}

The Arctic is portrayed in current climate models as a region of high sensitivity and widespread influence. State-of-the-art numerical models of the earth's climate system (each of which contains sophisticated modules for the ocean, atmosphere, cryosphere, and land surface) ${ }^{1}$ indicate that the surface warming associated with increasing levels of greenhouse gases in the earth's at-

\footnotetext{
${ }^{1}$ Hereafter, we refer to these models as general circulation models, or GCMs.

Corresponding author address: Dr. David S. Battisti, Department of Atmospheric Science, University of Washington, P.O. Box 351640, Seattle, WA 98195-1640.

E-mail: david@atmos.washington.edu
}

mosphere is greatest in the Arctic. However, little has been reported about the ability of these models to simulate the natural low-frequency variability in the arctic climate system, which, in reality, may obscure greenhouse gas warming.

Sea ice thickness varies on much longer timescales than the atmospheric forcing on the sea ice. Hence, variability in the arctic sea ice thickness may be used to measure the low-frequency variability in the arctic climate system. Recently, Bitz et al. (1996) used a model of the arctic atmosphere-sea ice-upper-ocean climate system (the polar cap climate model, or PCCM, described in section 2a) to examine the natural variability of the climate system, averaged over the arctic polar cap. Variability in the arctic climate stems from variability in the transport of energy into the Arctic by atmospheric circulation (see, e.g., Nakamura and Oort 1988). The results of Bitz et al. (1996), encapsulated in 

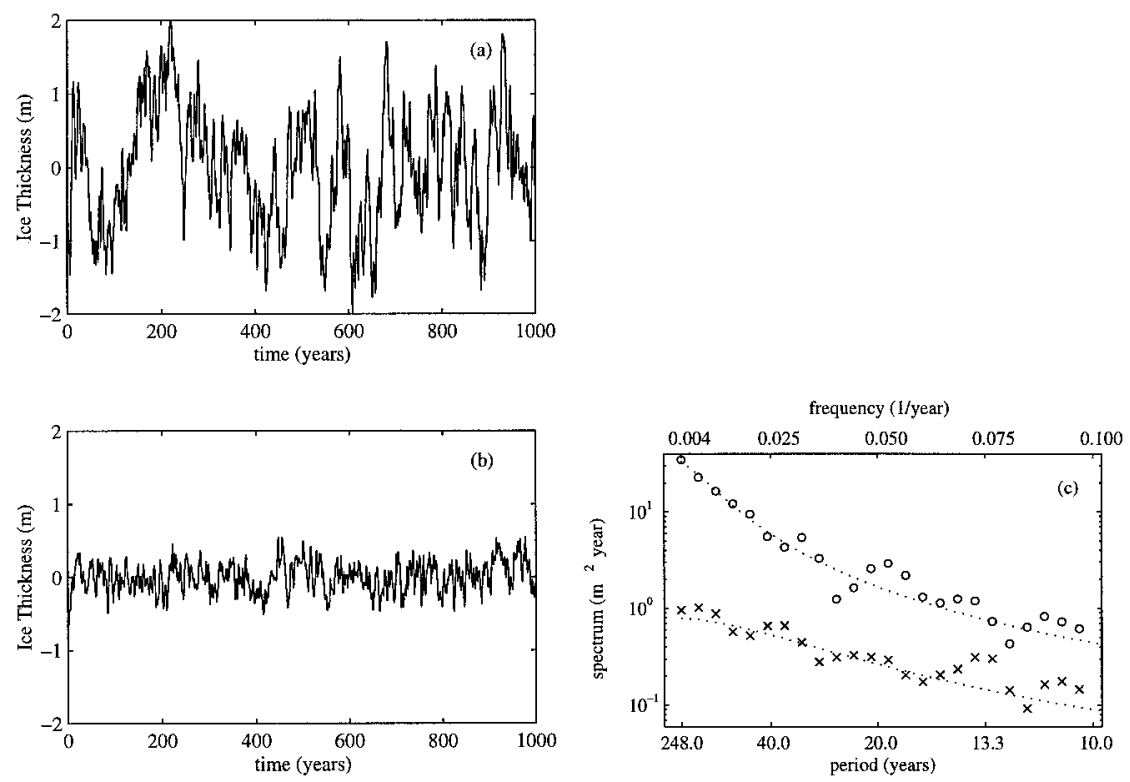

FIG. 1. One-thousand-year time series of the annual mean sea ice thickness anomaly averaged over perennial ice area in the Arctic from (a) the PCCM and (b) the GFDL climate model. Spectra (c) of the ice thickness from PCCM $(\bigcirc)$ and GFDL $(\times)$, each with a best fit (dotted line) to a discrete first-order Markov process. The spectra are calculated for the last $900 \mathrm{yr}(N)$ of model output using a lead/lag correlation method smoothed with a $124 \mathrm{yr}(L)$ Tukey window, yielding $8 N / 3 L=19$ degrees of freedom (Jenkins and Watts 1968).

Fig. 1a, indicate that variance in the arctic climate system occurs on timescales longer than a decade and at remarkably large amplitude (overall standard deviation in yearly ice thickness of $0.85 \mathrm{~m}$ ). This result is consistent with the calculations presented in Häkkinen and Mellor (1990) using a sea ice model forced with observed surface atmospheric conditions, and the inter-

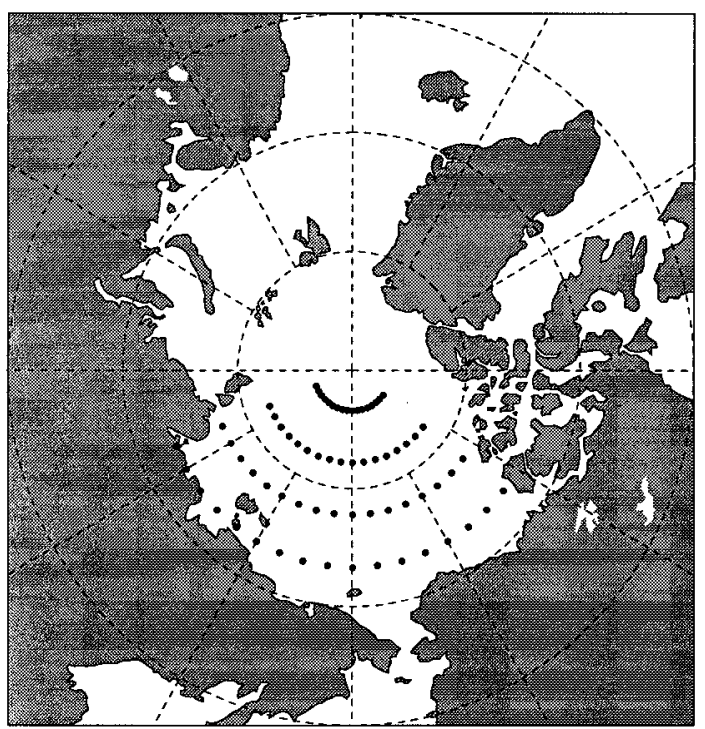

FIG. 2. Grid points from the GFDL model area that are used for comparison with the PCCM model output. This region is chosen because the sea ice is predominantly perennial. annual changes in the springtime ice thickness of order $1 \mathrm{~m}$ at the North Pole from a dozen submarine voyages, reported in McLaren et al. (1994). Analysis of the ice draft from moored sonars in the Beaufort Sea indicates changes in the multiyear ice thickness of $1.5 \mathrm{~m}$ during the period 1990 to 1995 (R. Moritz 1996, personal communication). Nonetheless, there are too few observational data to determine whether the climate variability that is demonstrated by the model of Bitz et al. (1996) is consistent with that in nature. We can, however, compare the results in Fig. 1a from the PCCM to that realized in a global climate model that includes a comprehensive treatment of each component of the hydrologic cycle. In Fig. 1b we show a time series of the anomalies of the mean annual arctic sea ice thickness for the area identified in Fig. 2 from a 1000-yr, "present climate" integration of the Geophysical Fluid Dynamics Laboratory (GFDL) GCM (Manabe and Stouffer 1996).

The amplitude of the simulated climate variability, evidenced here by the sea ice thickness in the GCM, is fundamentally different from that in the PCCM. The standard deviation for the annual sea ice thickness is $0.20 \mathrm{~m}$ for the GFDL GCM and $0.85 \mathrm{~m}$ for the PCCM, though the power spectrum of sea ice thickness (Fig. 1c) from the GFDL GCM and the PCCM indicate both models exhibit greatest variance in the sea ice thickness at low frequencies (see the appendix; Bitz et al. 1996). The difference in the natural variability depicted in Fig. $1 \mathrm{a}$ and that in Fig. 1b strongly affects the number of years one would have to monitor ice thickness to 
detect a climate change of 1-2 $\mathrm{m}$, as simulated by most GCMs in response to a doubling of $\mathrm{CO}_{2}$ (see, e.g., Bitz et al. 1996, 407).

Does the GFDL GCM underestimate the variability in the sea ice, or is the area-averaged ice thickness from the PCCM model in error? There are insufficient observations to answer this question definitively. In this study we will determine why there are large differences in the arctic climate simulated by the two models. In the process, we will obtain some insight concerning which of the two estimates in Fig. 1 is a more reliable estimate of the variability in the arctic climate system.

In this study we perform multiple, 1000-yr integrations of the PCCM to evaluate the sensitivity of the coupled climate system to the formulations of ice physics and to atmospheric forcing. We present results in which we modify the (standard) sea ice module in the PCCM to be identical thermodynamically to the sea ice module that is in the GFDL climate model. We then perform integrations in which we independently exchange several of the oversimplified physical formulations in the GCM ice module for the more realistic treatment that is used in the standard version of the PCCM; ultimately, we arrive back at the formulation of sea ice that is used in the standard version of the PCCM. We will demonstrate that adding realism to the parameterization of ice physics and increasing the resolution of temperature structure in the sea ice will lead to a substantial increase in the low-frequency variability in the arctic climate simulated by the GFDL GCM and other climate models. ${ }^{2}$

A brief overview of the PCCM and of the physical formulation of the sea ice in the GFDL general circulation model is found in section 2. Section 2 also contains a discussion of the observed flux of moist static energy into the Arctic, which is important for variability in the arctic climate and provides an important component in the forcing of the PCCM. Differences in this energy flux realized from the GCM and that from the observational record are identified, and in section 3 we examine the effect of these differences on the simulated variability in the arctic climate system using the standard PCCM. In section 4, we incrementally alter the PCCM to include several physical formulations in the sea ice module that are presently in the GFDL GCM and illustrate the sensitivity of the natural variability that is simulated in the model to these formulations. An estimate of the variability in the sea ice in nature is provided in section 5 , and a summary and discussion are presented in section 6 .

\footnotetext{
${ }^{2}$ We use the output from the GFDL model in this study because it is available and widely recognized. Other GCMs employ similar simplifications to sea ice, though details differ.
}

\section{Description of the models}

\section{a. The arctic polar cap climate model}

The area-averaged model of the arctic polar cap climate system is a one-dimensional coupled atmospheresea ice-surface ocean model of the arctic climate, averaged over the polar cap; hereafter, we refer to this model as the PCCM. The PCCM is forced by insolation, poleward atmospheric energy flux convergence, cloudiness, and snowfall. Natural climate variability in the PCCM occurs in response to the prescribed variability in the atmospheric energy flux convergence over the polar cap, $D$ :

$$
\begin{gathered}
D(n \tau)=\left(\int c_{p}[\overline{v T}] \frac{d p}{g}+\int L[\overline{v q}] \frac{d p}{g}\right. \\
\left.+\int[\overline{v \Phi}] \frac{d p}{g}\right) l / A,
\end{gathered}
$$

where

$$
[()]=\frac{1}{2 \pi} \int_{0}^{2 \pi}() d \lambda
$$

denotes a zonal mean and

$$
\left(^{-}\right)=\int_{0}^{\tau}() \frac{d t}{\tau}
$$

denotes a monthly mean ( $\tau=1$ month; $n$ is an integer). The terms on the right-hand side of Eq. (1) represent the fluxes of sensible heat, latent heat, and potential energy across a latitude circle with circumference $l$. The energy flux convergence is assumed to be distributed uniformly over the polar cap of area $A$ [see Overland and Turet (1994) and Bitz et al. (1996) for further discussion].

The sea ice model includes prognostic equations for sea ice thickness, snow depth, and temperature within the sea ice and at the upper surface. The sea ice is modeled as a horizontally uniform slab with no leads. The number of sea ice layers may be varied to examine the response of the system to changes in the resolution of the sea ice temperature profile. The surface albedo in the PCCM depends explicitly on the depth of the accumulated snowfall, and the GFDL surface albedo approximates the effects of snow accumulation with a parameterization based on the sea ice upper surface temperature.

The PCCM atmospheric model includes a sophisticated treatment of radiation, turbulent heat exchange at the sea ice surface, and a simple convective adjustment scheme. The atmosphere model has 18 vertical layers and is based on MacKay and Khalil (1991). Cloudiness and cloud height are prescribed stochastically. The upper ocean is modeled as a $50-\mathrm{m}$ slab mixed layer. The ocean temperature is fixed at the freezing temperature when there is sea ice. For further details of the model and demonstrations of the mean annual cycle and natural 
variability of the climate model, please see Bitz et al. (1996).

\section{b. Geophysical Fluid Dynamics Laboratory General Circulation Model}

The GFDL GCM is a global coupled atmosphere-sea ice-ocean model. The model physics are described in Manabe et al. (1992). Here, we review only the treatment of arctic sea ice. The ice is a slab of uniform thickness in each grid box without leads. Snow accumulation is converted immediately to equivalent sea ice thickness; therefore the thermal insulating capacity of snow is neglected and the surface albedo does not depend explicitly on snow depth. Heat conduction through the sea ice is calculated by assuming a linear temperature profile between the top and bottom surfaces of the ice; hence, sea ice is treated as a single layer with zero heat capacity (i.e., changes in the sea ice temperature require no energy). The sea ice is advected with the surface current, unless ice thickness exceeds $4 \mathrm{~m}$.

To mimic the effects of snow cover, melt ponds, and open water, the GFDL GCM surface albedo is specified as a function of the surface temperature,

$$
\alpha^{*}=\left\{\begin{array}{lc}
\alpha_{\text {ice }}, & \text { when } T_{s} \geq T_{m} \\
\alpha_{\text {ice }}+0.025\left(T_{m}-T_{s}\right), & \text { when } T_{m}-10^{\circ} \mathrm{K} \\
& <T_{s}<T_{m} \\
\alpha_{\text {snow }}, & \text { when } T_{s} \leq T_{m}-10^{\circ} \mathrm{K},
\end{array}\right.
$$

and sea ice thickness,

$$
\alpha_{G F D L}= \begin{cases}\alpha^{*}, & \text { when } h \geq 1 \mathrm{~m} \\ \sqrt{h}\left(\alpha^{*}-\alpha_{\text {ocean }}\right)+\alpha_{\text {ocean }}, & \text { when } h<1 \mathrm{~m} .\end{cases}
$$

In Eq. (2), $h$ is the sea ice thickness in meters, $T_{s}$ is the upper surface temperature, $T_{m}$ is the upper surface melting temperature, $\alpha_{\text {ice }}=0.55, \alpha_{\text {snow }}=0.8$, and $\alpha_{\text {ocean }}=$ 0.1 .

\section{Discrepancies due to atmospheric forcing in the arctic region}

The natural variability in the Arctic is rooted fundamentally in the variability of energy transported by the atmosphere into the Arctic. Natural variability in the climate of the polar cap modeled by the PCCM is a response to the prescribed, time-dependent atmospheric energy flux convergence, $D$, and cloudiness (Bitz et al. 1996). Could the discrepancy in the sea ice variability in the two models (see Fig. 1a and 1b) be due to the differences in the energy transport to the Arctic that is realized in the GFDL GCM and the energy transport that is prescribed (based on observations) in the PCCM? To answer this question, we will compare PCCM simulations forced by (i) $D$ based on observations and (ii) $D$ from the GFDL GCM.

\section{a. Atmospheric energy transport into the Arctic in nature and in the GFDL GCM}

The observed monthly values of $D$ for a polar cap bounded by $70^{\circ} \mathrm{N}$ are computed by Overland and Turet (1994) based on rawinsonde data from 1965 to 1989. The values of $D$ from the GFDL GCM output are computed as a residual from the energy budget of the polar cap (see Nakamura and Oort 1988),

$$
\Delta E / \Delta t=F_{o}+D+F_{s},
$$

where $\Delta E / \Delta t$ is the rate of storage of energy in the atmosphere, $F_{o}$ is the net incoming flux of shortwave and longwave radiation at the top of the atmosphere, and $F_{s}$ is the upward energy flux at the earth's surface. ${ }^{3}$ The GFDL model output is given as monthly mean values. Here, $\Delta E / \Delta t, F_{o}$, and $F_{s}$ are computed at each grid point and then averaged over all of the grid points shown in Fig. 2. Therefore, $D$ represents the monthly poleward transport of energy across the boundary of the area covered by the grid points. ${ }^{4}$

Figures $3 a$ and $3 b$ show the mean annual cycle and the standard deviation of $D$ from observations and GFDL model output. The mean monthly $D$ from the GCM is $5 \%-10 \%$ larger than the observed $D$ in all months except November through January. The standard deviation of monthly $D$ from the GFDL GCM is about $60 \%$ of observed during the winter and about $80 \%$ of observed during the melt season. The power spectrum and autocorrelation for the observed and GFDL-derived monthly anomaly of $D$ are shown in Figures $3 \mathrm{c}$ and $3 \mathrm{~d}$. The power spectrum of the observation-based $D$ is nearly constant for monthly and longer timescales, while the variance in $D$ from the GFDL model output is skewed toward short timescales (at least as short as 2 months). The power spectrum of $D$ from the GFDL model output is similar to observations for monthly timescales, but it is considerably lower for timescales greater than about 5 months (see also section 5).

To examine the sensitivity of the climate variability in the Arctic requires long integrations (e.g., $1000 \mathrm{yr}$ each) because of the long timescale associated with the thermodynamic adjustment of sea ice. The observed time series of $D$, however, spans less than $30 \mathrm{yr}$. In addition, only the mean monthly $D$ is available from both observations and the GFDL GCM, though $D$ probably varies considerably on shorter timescales. Hence,

\footnotetext{
${ }^{3}$ Here, $D$ is not directly calculable from the information that is saved from the GCM integration.

${ }^{4}$ The areas used to calculate $D$ from the model and the observations differ because the region that is most appropriate for input to the area-averaged model (i.e., over the perennial arctic sea ice) is void of observations with which to calculate the observed $D$. We note, however, in the GFDL model $D$ along $70^{\circ} \mathrm{N}$ is less than $D$ over the region of perennial sea ice, which suggests the observations at $70^{\circ} \mathrm{N}$ are likely to provide an underestimate of the true $D$ over the perennial sea ice.
} 

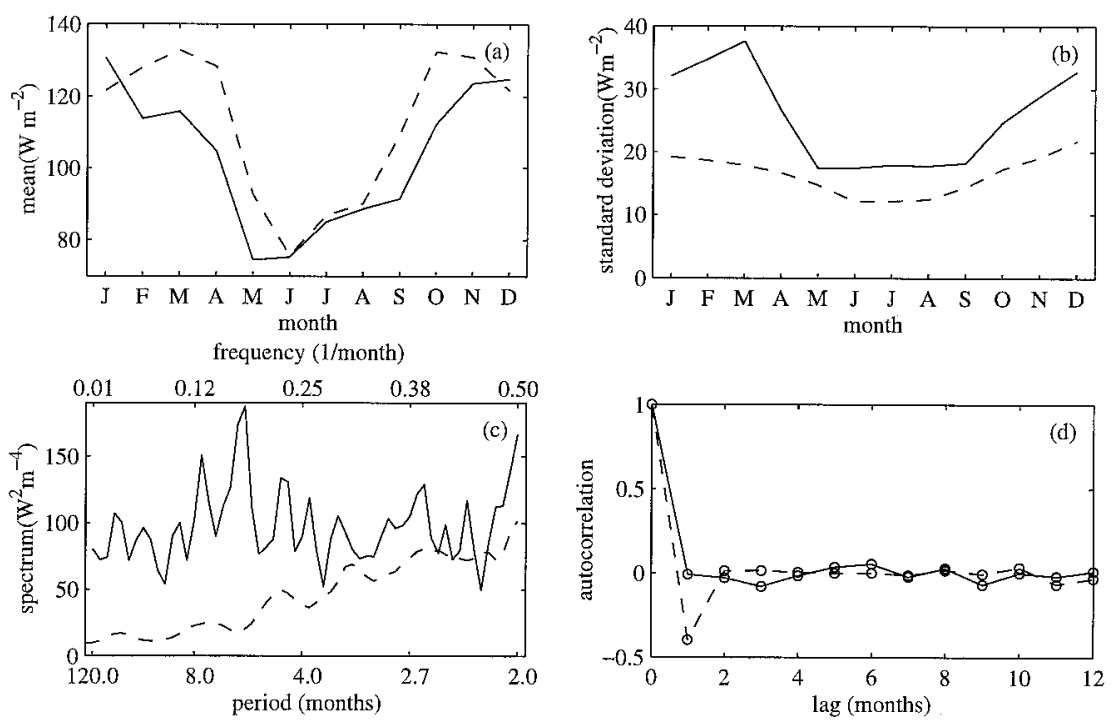

FIG. 3. The mean monthly atmospheric energy flux convergence: (a) annual cycle, (b) standard deviation, (c) power spectrum, and (d) autocorrelation. Data are presented for both observations (solid line) and the GFDL model (dashed line). The power spectra are calculated as in Fig. 1c, but here the annual cycle is removed and the window width is 4 yr. Hence, there are 600 (16) degrees of freedom in the GFDL output (observations).

to force the PCCM with a 1-day time step, synthetic time series of $D$ must be obtained for both the observations and the GCM.

Based on observations, we obtain a synthetic $D$ from a discrete form of the Langevin equation (Reif 1965, 560) so that the standard deviation and power spectrum of monthly mean values are consistent with those in Figs. $3 \mathrm{~b}$ and $3 \mathrm{c}$, and the power spectrum of the daily time series of $D$ is red with a characteristic timescale of 5 days (see Bitz et al. 1996). Based on GFDL output, again, we simulate $D$ from a discrete form of the Langevin equation; however, now the daily time series is partially high-pass filtered to render the synthetic spectrum commensurate with that from the GFDL model.

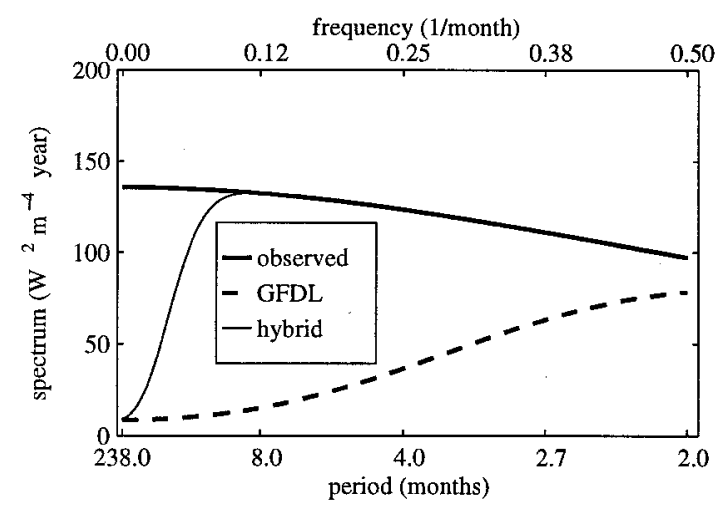

FIG. 4. Spectra of synthetic $D$ based on observations (heavy solid) and the GFDL GCM (heavy dashed). Also plotted is the spectrum of the hybrid $D$ (light solid), discussed in section 5. All curves are computed analytically from the difference equations and spectral filters used to synthesize $D$.
Power spectra of the synthetic time series are shown in Fig. 4; the spectral properties of the synthetic $D$ are statistically indistinguishable from their observed counterparts in Figs. $3 \mathrm{c}$ and $3 \mathrm{~d}$.

\section{b. Sensitivity of the ice thickness variability to differences in $D$}

We first demonstrate that the area-averaged PCCM model reproduces the sea ice variability simulated by the GFDL GCM. The sea ice module in the PCCM is replaced with the thermodynamic treatment of sea ice that is identical to that in the GCM. The modified PCCM was able to reproduce a significant portion of the lowfrequency variability in the GFDL 1000-yr time series of sea ice that is shown in Fig. 1b when forced by the accompanying $D$ taken directly from the GFDL climate model. Specifically, about $45 \%$ of the sea ice variance in the GFDL control integration is accounted for in the hindcast (not shown) even though we have neglected the ocean heat transport variability and used only monthly averaged values of $D$ from the GCM using a cubic spline to produce a daily time series. As a second test, a 1000-yr integration is performed while forcing the modified PCCM with the GFDL synthetic $D^{5}$ (Fig. 4). A 300-yr segment of the time series of the sea ice thickness from this model is displayed in Fig. 5c. The standard deviation $(\sigma)$ of the yearly sea ice thickness

\footnotetext{
${ }^{5}$ Henceforth, when we refer to forcing of the PCCM with the GFDL GCM $D$ or the observed $D$, we are referring to the synthesized $D$ that are described at the end of section 3a.
} 


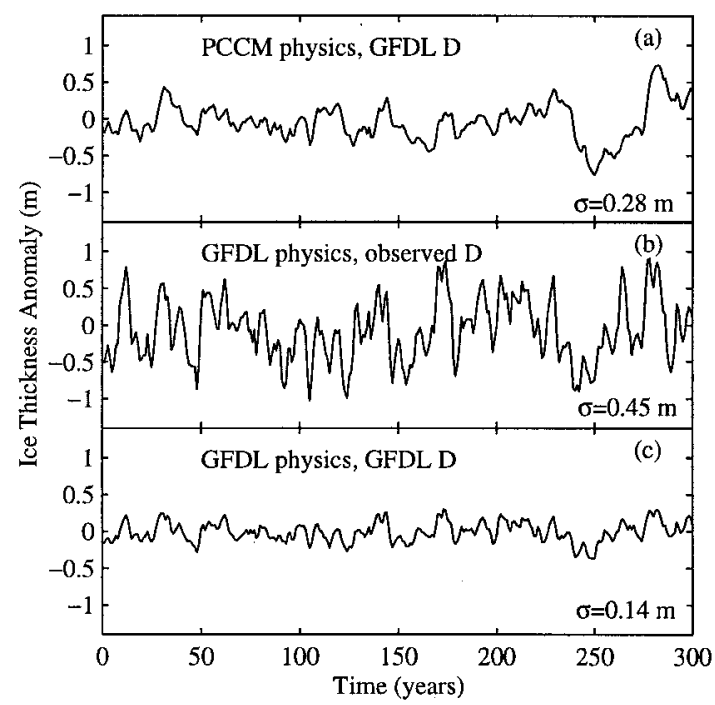

FIG. 5. Anomalies of yearly sea ice thickness from the PCCM output for integrations with (a) $D$ based on GFDL, standard PCCM surface physics for snow and albedo, six sea ice layers; (b) $D$ based on observations, GFDL surface physics for snow and albedo, one sea ice layer; (c) $D$ based on GFDL, GFDL surface physics for snow and albedo, one sea ice layer.

from the PCCM with the GFDL GCM sea ice physics [e.g., albedo as per Eq. (2), one layer of ice, and no snow cover] is $\sigma=0.14 \mathrm{~m}$, similar to that from the $\operatorname{GCM}(\sigma=0.20 \mathrm{~m})$ and very different from that realized by the standard physics PCCM forced by the observed $D(\sigma=0.85 \mathrm{~m})$.

To examine how much of this remarkable difference in the sea ice variability can be attributed to the differences in the forcing, we reran the PCCM model with the sea ice physics of the GFDL GCM, but this time the forcing $D$ was prescribed based on the observations. This integration also yielded sea ice variability ${ }^{6}$ that is predominantly at low frequencies [Figs. 5b and 6 (heavy dashed line)] but with amplitude $(\sigma=0.45 \mathrm{~m})$ that is intermediate between the amplitude achieved in the standard physics PCCM forced by the observed $D(\sigma$ $=0.85 \mathrm{~m}$ ) and the amplitude realized by the PCCM with the GFDL GCM $D$ and the GCM ice physics ( $\sigma$ $=0.14 \mathrm{~m}$ ).

Finally, we integrated the standard physics PCCM, forcing it with the $D$ based on the GFDL GCM. The standard deviation is reduced to one-third $(0.28 / 0.85)$ of that from the integration using the observed $D$; the variance remains in the lower frequencies [Figs. 5a and 6 (heavy solid line)].

In summary, the results of these four PCCM integrations (see Table 1) demonstrate the following:

- When the PCCM is modified to contain the GFDL GCM thermodynamic sea ice package and is forced

\footnotetext{
${ }^{6}$ Variability is used synonymously with standard deviation.
}

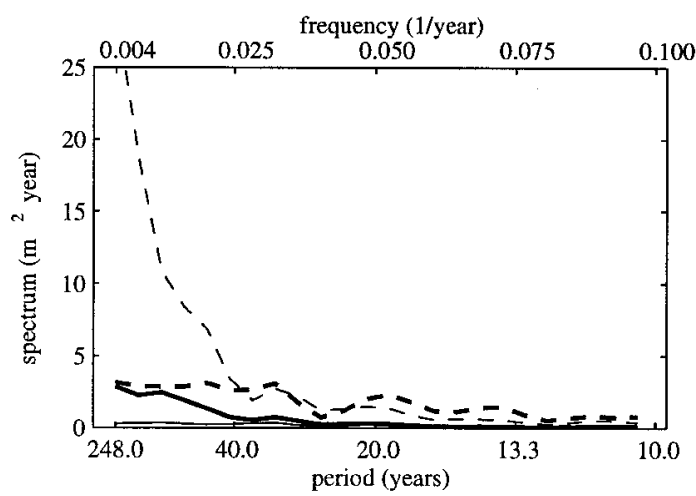

FIG. 6. Power spectra of yearly sea ice thickness anomalies from the PCCM output for integrations: $D$ based on GFDL, standard surface physics for snow and albedo, six sea ice layers (heavy solid); D based on observations, GFDL surface physics for snow and albedo, one sea ice layer (heavy dashed); D based on GFDL, GFDL surface physics for snow and albedo, one sea ice layer (light solid); the hybrid $D$ and the PCCM standard physics (light dashed). The power spectra are calculated as in Fig. 1c.

by the energy transport that is realized by the GCM, the variability in the sea ice is statistically very similar to the sea ice variability from the full GCM;

- The variability in arctic sea ice in the area-averaged (PCCM) regional climate model is substantially affected by the different formulations of the sea ice physics (see section 2), and by the differences between the energy transport into the Arctic realized by the GFDL GCM and that which is observed in nature.

\section{Sensitivity studies reveal the fundamental physics that accounts for the quiescent arctic climate in the GCM}

The experiments of the last section showed that the sea ice variability in the PCCM almost doubles ${ }^{7}$ when the simple module for sea ice from the GCM (section 2 ) is replaced by the more realistic formulation in the standard PCCM, described in section 2a (and used in Bitz et al. 1996). In this section we will isolate aspects of the sea ice treatment that significantly affect the variability in the sea ice and in the arctic climate system that is simulated by the PCCM.

The two fundamental differences between the ice models used in the PCCM and GCM are the following.

1) GFDL GCM: The surface physics do not resolve snow cover. The albedo parameterization depends on the upper surface temperature and sea ice thickness but does not depend on snow depth explicitly. In-

\footnotetext{
${ }^{7}$ The increase in the standard deviation of annual mean ice thickness due to the inclusion of a more realistic sea ice module is 0.28 / $0.14=2$ when the PCCM is forced by the GCM $D$ and $0.85 / 0.45$ $=1.9$ when the PCCM is forced by the observed $D$.
} 
TABLE 1 . The standard deviation of yearly sea ice thickness anomalies for four integrations of the PCCM described in section 3b. Each integration uses a unique permutation of the forcing $(D)$ and treatment of sea ice.

\begin{tabular}{lcc}
\hline \hline \multicolumn{2}{c}{ Experiments with different sea ice physics packages } \\
\hline & $\begin{array}{c}\text { GCM (GFDL) } \\
\text { ice physics }\end{array}$ & $\begin{array}{c}\text { PCCM } \\
\text { ice physics }\end{array}$ \\
\hline$D$ as per GFDL GCM & $0.14 \mathrm{~m}$ & $0.28 \mathrm{~m}$ \\
$D$ as per observations & $0.45 \mathrm{~m}$ & $0.85 \mathrm{~m}$ \\
\hline
\end{tabular}

solation effects and energy storage in snow cover are excluded.

PCCM: The snow depth is treated as a prognostic variable in the sea ice model. The surface albedo parameterization reflects the sea ice thickness and the snow depth. The surface is insulated by accumulated snow cover, and energy is required to melt it.

2) GFDL GCM: The vertical temperature profile is constrained to be uniformly linear. Hence, the sea ice model has one layer.

PCCM: The temperature profile is well resolved by six sea ice layers.

Table 2 summarizes the results from the $(1000 \mathrm{yr})$ integrations of the PCCM using all possible permutations of the forcing (GFDL GCM or observed), sea ice resolution (one layer [GFDL GCM), or six layers (standard)], and snow/albedo formulation (see section 2). The quartet of experiments on the left (right) summarizes results for varying sea ice resolution and the treatment of snow/albedo while keeping the forcing $D$ prescribed as in the GCM (from observations).

For forcing by the observed $D$ and with the treatment of snow/albedo as in the GFDL GCM, increasing the number of layers in the sea ice from a purely linear $(n$ $=1$ ) profile to $n=6$ layers (hence better resolving conduction) causes an increase of $27 \%$ (0.45 to $0.57 \mathrm{~m})$ in the standard deviation of sea ice thickness; using the standard PCCM albedo/snow formulation yields an increase in the variability of $49 \%(0.57$ to $0.85 \mathrm{~m})$. The percent increase of the sea ice variability associated with the adequate resolution of conduction depends on the treatment of snow/albedo, yet it is relatively insensitive to the prescription of $D$ (compare rows in Table 2).

For forcing by the observed $D$, changing the snow/ albedo formulation from the GFDL GCM formulation (Eq. 2) to the explicit treatment in the standard version of the PCCM leads to an increase in the standard deviation of sea ice thickness of $27 \%(0.45$ to $0.57 \mathrm{~m})$ with $n=1$ and $49 \%$ ( 0.57 to $0.85 \mathrm{~m})$ with $n=6$. Again, the percent increase of the sea ice variability resulting from improving the formulation of snow/albedo is relatively insensitive to the prescription of $D$ (compare columns in Table 2).

In summary, either increasing the resolution of the temperature profile in the sea ice or implementing the
TABLE 2. The standard deviations of yearly sea ice thickness anomalies from eight integrations, which differ by the number of sea ice layers, $n$, and the surface physics, which either explicitly include the effect of snow or (as in the GFDL model) there is no snow cover and the albedo is described by Eq. (2). The integrations in the left (right) quartet were forced with the variability in $D$ that is based on GFDL model output (the observed data). The schematic indicates the percent change in standard deviation as one moves through the quartet of experiments, using either prescription of $D$.

\begin{tabular}{|c|c|c|c|c|}
\hline & \multicolumn{2}{|c|}{ GFDL $D$} & \multicolumn{2}{|c|}{ Observed $D$} \\
\hline & $n=1$ & $n=6$ & $n=1$ & $n=6$ \\
\hline \multirow{5}{*}{$\begin{array}{l}\alpha_{\text {surface }}=\text { GFDL } \\
\alpha_{\text {surface }}=\text { explicit }\end{array}$} & \multirow{2}{*}{$\begin{array}{l}0.14 \mathrm{~m} \\
0.19 \mathrm{~m}\end{array}$} & $0.18 \mathrm{~m}$ & \multirow{2}{*}{$\begin{array}{l}0.45 \mathrm{~m} \\
0.57 \mathrm{~m}\end{array}$} & \multirow{3}{*}{$\begin{array}{l}0.57 \mathrm{~m} \\
0.85 \mathrm{~m}\end{array}$} \\
\hline & & $0.28 \mathrm{~m}$ & & \\
\hline & \multicolumn{2}{|c|}{$\stackrel{130 \%}{\longrightarrow}$} & \multirow{3}{*}{$150 \%$} & \\
\hline & \multirow[t]{2}{*}{$130 \%$} & \multirow[b]{2}{*}{$\sim 190 \%$} & & \\
\hline & & & & \\
\hline & & $\overrightarrow{150 \%}$ & & \\
\hline
\end{tabular}

improved snow/albedo formulation from that in the GFDL GCM, independently, leads to a substantial increase in sea ice variability in the regionally averaged arctic climate (PCCM) model. Implementing these improvements simultaneously causes the variance to increase by about fourfold, independent of the different prescriptions of forcing $D$ (see footnote 7 ).

\section{An estimate of the variability of sea ice thickness in nature}

The natural variability in the arctic climate system is driven by changes in the energy transport into the Arctic by the atmosphere $(D)$ and by the ocean. We have shown that the differences between the atmospheric energy transport in the GFDL GCM and that in nature (Figs. $3 \mathrm{c}$ and 4 ) have a large impact on the variability of the arctic climate in the PCCM (e.g., a factor 0.85/0.28 = 3 difference in the standard deviation of sea ice thickness; Table 1). Hence, to obtain a reliable estimate for the amplitude of the natural variability in the arctic climate system requires that $D$ be known accurately on a wide range of timescales.

For periods of less than a few years, the differences in $D$ from the observations and from the GFDL model are statistically significant (based on sample size) and are too large to be attributed to observational error. In contrast, for periods greater than about $5 \mathrm{yr}$, the observational record is too short to determine the true spectrum of $D$ : significant uncertainties are introduced from both measurement and sampling error. However, we may anticipate that in the decadal range, nature has at least as much variability in $D$ as does the GFDL model. ${ }^{8}$

\footnotetext{
${ }^{8}$ The interannual to multidecadal variability in the global Tropics of the GFDL GCM is anemic compared to nature (Lau et al. 1992); one may expect that this problem is communicated via atmospheric
} dynamics to the polar regions. 
TABLE 3. As in Table 2 but the forcing $D$ has spectral characteristics that represent a hybrid of the spectra from the GCM and from observations.

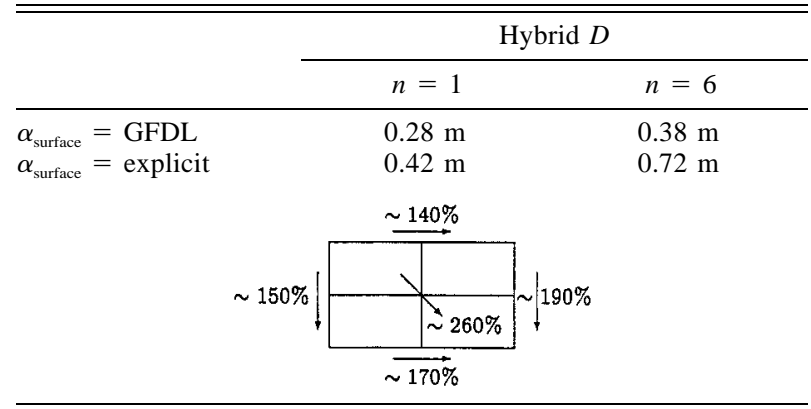

To obtain a conservative "best estimate" of the sea ice variability in the arctic climate system, we assemble a hybrid $D$ that has spectral properties that are consistent with the observations on timescales shorter than $2 \mathrm{yr}$, and is intermediate between the $D$ from the GFDL model and from observations for timescales longer than 2 yr. This hybrid spectrum is plotted in Fig. 4 along with the spectra based on the GFDL and observed $D$.

When the standard physics PCCM model is forced by this hybrid $D$, the variability in the sea ice thickness still accumulates at the lowest frequencies (Fig. 6, light dashed line) and the overall variability is $0.72 \mathrm{~m}$ - only slightly reduced from that obtained using the PCCM and the full-spectrum observed $D(0.85 \mathrm{~m})$. Thus, the best estimate of the standard deviation due to natural variability in sea ice is more than a factor of $3(0.72 /$ $0.20)$ greater than that simulated by the GCM. The remaining experiments with the PCCM using the hybrid $D$ and various mixes of sea ice physics are summarized in Table 3. Using the hybrid $D$, the sensitivity of the simulated sea ice variability to specific changes in the treatment of sea ice is consistent with that obtained using the observed and GFDL $D$ (contrast rows and columns between the two tables, Tables 2 and 3).

We have not examined the impact of sea ice dynamics or leads on the variability in the arctic climate system. Bitz et al. (1996) present a calculation that suggests the uniform advection of sea ice acts to dampen slightly the variability displayed in the standard physics PCCM (Fig. 1). However, the (observed) climatological mean advection of sea ice ensures that the thickest sea ice is nearly collocated with the region of maximum variability in the energy transport $D$ (north of Greenland), which will significantly amplify the variability in the sea ice volume from that in our regionally averaged model (Bitz et al. 1996). It is unknown to what extent the variability in the mechanical forcing (wind and current stresses) of the sea ice affects the low-frequency variability in the arctic climate system. We anticipate, however, that variability in the ocean heat transport, which is neglected in this study, will increase the variability in the sea ice from the best estimate presented here that is due (solely) to the stochastic nature of the atmospheric energy transport.

\section{Conclusions and discussion}

In this paper we have used a model of the area-averaged, coupled arctic atmosphere-sea ice-ocean system to examine the variability in the arctic climate that may be expected because of the natural variability in the transport of energy into the Arctic via the atmospheric circulation. We use sea ice thickness as a useful indicator of the state of the arctic climate system.

The motivation for this work stems from the results of the modeling study of Bitz et al. (1996), who found that the variability in the arctic climate was surprisingly large and predominantly at multidecadal and longer timescales. Though the scant observations are not inconsistent with the model results, the observational record is too short to determine the veracity of this result. Hence, we contrasted the PCCM results to that found in a global general circulation (climate) model, and found that the variability in the sea ice of the GCM was one-quarter of that in the PCCM (see section 1). In this study, we have used the PCCM diagnostically to help determine the reasons for the qualitative differences between the PCCM and the arctic climate simulated by the GFDL GCM, and summarize the reasons below. Some of the results from the present study are relevant to most—if not all —of the GCMs in use today to study natural climate variability and the climate change associated with increasing greenhouse gases; they suggest that the variability in the arctic climate is probably much more energetic than is being simulated in these general circulation (climate) models [see sections $6 \mathrm{a}$ and $6 \mathrm{~b}(2)$ below].

\section{a. Arctic sea ice variability in the GFDL General Circulation Climate Model}

We inserted the GFDL sea ice physics into the PCCM and then forced the model with the synthetic energy transport that is statistically identical to that in the GFDL GCM (section 3b). The sea ice thickness variance is similar to that from the "present climate" integration of the GFDL climate model. Thus, results that we have presented concerning the impact of improving the sea ice treatment on the variability in the arctic climate are likely to be quantitatively relevant to the GFDL climate model. ${ }^{9}$

\footnotetext{
${ }^{9}$ It is unlikely that differences in the details of the GFDL and PCCM atmosphere models within the Arctic would render the PCCM model not applicable to the GCM. Bitz et al. (1996) found the sea ice thickness variability is relatively insensitive to the details of the stochastic cloud forcing; correlating perturbations in the cloud forcing with the atmospheric heat flux convergence has a modest effect on the model variability.
} 
Our results suggest that the GFDL GCM will exhibit substantially more natural variability in the arctic climate system (e.g., a factor of 4 in sea ice variance) if unarguable improvements are made in the treatment of sea ice in the model (see section 4). Furthermore, there is a fundamental difference in the variability in the sea ice thickness displayed in the PCCM driven by the atmospheric energy transport $D$ from the GFDL model and that driven by the observed $D$ : the standard deviation of the sea ice in the PCCM with the GCM $D$ is about one-third of that from the model forced with the observed $D$ (see section $3 \mathrm{~b}$ ). Combining the improvements in the treatment of the sea ice physics with (unidentified) changes to the GCM so that the atmospheric energy transport into the Arctic is consistent with that observed, our calculations indicate that the variance in the arctic sea ice simulated by this GCM will increase by a factor of 10 !

The variance of the atmospheric energy transport in the GFDL GCM is less than that from observations on all timescales (Fig. 3). Most important for the variability in the arctic climate system, the GFDL GCM severely underrepresents the variability on the seasonal to interannual timescales-the timescales at which the observed energy transport is accurately determined. The literature indicates this is a problem ubiquitous to lowresolution models [see, e.g., Held and Phillipps (1993) and Boyle (1993) for a discussion of the resolution dependence of energy transport in the synoptic band], and herein lies a fundamental problem with using a GCM to examine the variability in the arctic climate system: the significant variability in the arctic sea ice thickness is on multidecadal timescales and it is sensitive to the amplitude of the variability in the meridional energy transport by the atmosphere into the Arctic at all frequencies but particularly to the amplitude of the highfrequency variability (Bitz et al. 1996). Thus, to study the natural variability in the arctic climate with a GCM will require multiple very long (multicentury) integrations at high resolution (at least T42), which is not feasible on today's hardware.

\section{b. Arctic sea ice variability simulated by other models}

In this section, we compare the sea ice variability demonstrated by the PCCM with results from other models. Two other classes of models have been used to assess the natural variability in the arctic sea ice: (a) sea ice-upper-ocean models of the Arctic, and (b) full climate models that couple an atmospheric GCM to a sea ice model and a model of the oceans. The class (a) models require one to prescribe the historical record of forcing of the ice-ocean system at the surface of the sea ice; the data required to do this have only recently become of sufficient duration to determine this forcing over the Arctic (but there are insufficient data to determine the observed arctic ice volume at any time in the historical record). The results from the studies of Walsh et al. (1985), Häkkinen (1993), Flato (1995), and Häkkinen and Mellor (1990) using type (a) models are pertinent to our study. We note, however, that since the variability is likely to be dominated by multidecadal and longer timescales, for all of the model studies discussed below (excepting perhaps Häkkinen and Mellor 1990) the model integrations are too short to determine, even qualitatively, the amplitude of the variability in the arctic climate system.

\section{1) Coupled SEA ICE/OCEAN MOdels OF THE ARCTIC}

Häkkinen (1993) used a dynamic-thermodynamic sea ice model of the Arctic to study the freshwater export from Fram Strait for the period 1955-75. The prescribed forcing of the model was heat flux derived from the climatological mean air temperatures and the observed wind stress for that period. The variability in the simulated sea ice volume for this period was about $10 \%$ of the mean sea ice volume. Walsh et al. (1985) and Flato (1995) used different dynamic-thermodynamic sea ice models to perform hindcasts of the sea ice variability in the Arctic from 1951 to 1980 (Flato's hindcast extends to 1990). The standard deviation of the averaged sea ice thickness in these simulations is also much smaller than that obtained with the PCCM but comparable to that in Häkkinen (all three studies found variability greater than that from the GFDL climate model).

In the studies of Häkkinen (1993) and Flato (1995), the heat fluxes used to force the model are calculated using the bulk formula and the climatological mean surface air temperatures, ${ }^{10}$ which ensures an artificial, strong damping of the sea ice variability. ${ }^{11}$ In contrast, the sea ice in the PCCM experiences only anomalous thermodynamic (not dynamic) conditions, and these anomalies are affected by interactions between the atmosphere-sea ice-ocean system. We note that Häkkinen and Mellor (1990) performed a study of the arctic sea ice from 1880 to 1985 using a one-dimensional sea iceupper-ocean model. In this study, the forcing included the observed surface heat fluxes calculated (in part) from the observed surface atmospheric temperature anomalies. The sea ice variability displayed in this hindcast was consistent with that simulated by the PCCM (Häkkinen 1995, personal communication).

In summary, the results from these three studies suggest that thermodynamic processes are a major conduit

\footnotetext{
${ }^{10}$ The forcing of the sea ice model in Flato's study is largely confined to the observed wind stress anomalies: the model experienced interannually varying air temperatures only near the coasts.

${ }^{11}$ For analogous reasons there is an artificial reduction of the variance in the atmosphere when atmospheric GCMs are integrated using climatologically prescribed SSTs [see, e.g., Barsugli (1995) and Manabe and Stouffer (1996)].
} 
for affecting variability in the sea ice volume in the Arctic. In addition, the results do not contradict the expectation, based on the PCCM results, that the variability in the sea ice thickness may be a significant fraction of the mean.

\section{2) Global climate models}

There are about two dozen full climate models that are presently being used to study the variability in the global atmosphere-ocean-land-sea ice climate system. Apparently, the natural variability in the sea ice has been examined in only three of these models (this includes the documentation of the GFDL model in this study). In the Hadley Center climate model (HADCM2; Mitchell et al. 1994), the standard deviation in the sea ice volume is about $10 \%$ to $20 \%$ of the mean sea ice volume (T. Johns 1995, personal communication). The standard deviation in the sea ice volume from a $20-y r$ control integration of the CSIRO climate model is about $20 \%$ of the mean sea ice volume (Hunt et al. 1995). Unfortunately, the results from these studies are not directly comparable to that from the PCCM because our results do not allow for variability in the sea ice extent. A significant portion of the variability in the sea ice volume in these two GCMs is due to variability in the sea ice extent. Hence, it is likely that the variability in the sea ice thickness over perennial ice regions in these global climate models is much less than that displayed in the PCCM, and this is expected from the sensitivity studies using the PCCM in sections 4 and 3b. Specifically, the atmosphere component of the CISRO climate model discussed in Hunt et al. (1995) has low horizontal resolution (R15), which enables long integrations to be made but ensures a deleterious effect on the sea ice variability via distortions in the atmospheric energy transport (see section 3b). The HADCM2 model uses a moderate horizontal resolution in its atmosphere model, but has a one-level sea ice formulation, which, according to the results in section 4 , will artificially reduce (by about half) the variance in the sea ice from that which would be realized in the same climate model but with the conduction of heat in the sea ice is adequately resolved. Hence, we hypothesize that sea ice variability in the GFDL, HADCM2, and CSIRO climate models is significantly less than that in nature.

\section{c. Implications}

We have confirmed that in the regionally averaged model of the coupled atmosphere-sea ice-ocean arctic climate system, the natural climate variability is extremely sensitive to the formulation of the sea ice. Specifically, the variance in the sea ice simulated by the coupled model nearly doubles when snow is explicitly included in the model and a more realistic parameterization for the albedo of snow/ice is used in place of the standard formulation of albedo used in several
GCMs. When there is adequate resolution of the temperature gradient (i.e., the conductive flux) in the sea ice, again the sea ice variance nearly doubles. Implementing these improvements simultaneously causes the variance to increase by about fourfold. The result from increasing the temperature resolution may have been anticipated by combining the results from Semtner (1976) (concerning the sensitivity of the annual cycle of sea ice to the number of layers in the sea ice) with those from Bitz et al. (1996), who demonstrated that low-frequency variability in arctic climate is largely a result of nonlinear physics that are associated with sea ice that is undergoing both annual cycle forcing and stochastic (high-frequency) forcing.

There are two implications from these results for detecting an anthropogenically forced climate change in the Arctic. To detect a climate change, the amplitude and character of the natural variability (the noise) must be known. There are insufficient observations to document the natural variability in the arctic climate system [due to the presence of sea ice, the arctic climate system adjusts slowly (Thorndike 1992)]. Unfortunately, our results suggest that the treatment of sea ice in the climate models that are presently used to address the detection problem seriously jeopardizes the veracity of the simulated natural arctic climate variability. Furthermore, since the variability in the arctic climate is extraordinarily sensitive to the full spectrum of the variability in the meridional energy transport $(D)$ into the Arctic, it is important that these climate models simulate accurately all of the many processes that constitute $D$.

Second, climate models that are presently used to evaluate climate change due to increasing carbon dioxide suggest that the greatest changes will be in the mean arctic climate and inherently tied to changes in the sea ice thickness, particularly to large changes in the mean sea ice thickness. To our knowledge (G. Flato, J. Maslanik, J. Walsh, and J. Weatherly 1995, personal communications), excepting the two studies mentioned in the previous section $[6 b(2)]$ and the GFDL climate model, the natural variability of sea ice within the Arctic of these models has not been examined. However, because these global climate models have in common low horizontal resolution atmospheres (see, e.g., Houghton et al. 1990), the climate change simulated by these GCMs is likely to be large compared to the natural variability within the arctic in the control integrations of these climate models [e.g., the standard deviation of the area-averaged sea ice thickness is $0.20 \mathrm{~m}$ for the control integration of the GFDL climate model, whereas the change in the mean sea ice thickness associated with a doubling $\mathrm{CO}_{2}$ is typically more than $2 \mathrm{~m}$ (Manabe et al. 1991)]. In contrast, our results indicate that the natural variability within the arctic is much larger than that simulated by these models, and it may be a substantial fraction of the predicted change in mean ice thickness. Hence, the present estimate of the ratio "signal (climate change)" to "noise (natural variability plus observa- 
tional error)" is likely to be greatly overestimated in the Arctic. Finally, our results raise serious questions concerning the viability of the predicted equilibrium climate change using these models because of the strong nonlinearities and long timescales inherent to the variability in the Arctic (due to sea ice).

It is commonly stated that the arctic climate is sensitive to external (e.g., anthropogenic) forcing because of the feedback involving changes in the ice extent and the surface albedo. In light of our results, it is not surprising that the widest variations among different GCM simulations of the present mean climate, and of the climate response to greenhouse gas increase, occur in the Arctic (e.g., Houghton et al. 1990). Indeed, the differences in the annual cycle of the arctic surface temperature response simulated by different GCMs would represent enormous climate changes, with important implications both inside and outside the Arctic. Based on the results presented in this paper, our previous results (Bitz et al. 1996) and the results of others, it is likely that the wide variability of the arctic climate simulated by the GCMs result from the model formulations of arctic physical processes that determine the surface albedo, sea ice thickness, snow depth, and cloudiness. It is well known that these formulations are poorly matched to observed features of the arctic atmospheresea ice-ocean system, but relatively little attention has been paid to improving the formulations in ways that are relevant to the global climate problem. A recent summary of these issues may be found in the SHEBA Prospectus (Moritz et al. 1993).

Acknowledgments. We are grateful to S. Manabe, R. Stouffer, and the National Climatic Data Center for making the climate model data available to us, and to $\mathrm{G}$. Flato, S. Manabe, and N. Untersteiner for helpful discussions during the course of this work. The following graciously provided information on the arctic climate simulated by various (GCM) climate models: H. Cattle and T. Johns (HADCM2), J. Maslanik (GENESIS), and J. Weatherly (NCAR). We thank K. A. Runciman for calculating the atmospheric heat flux convergence and the area-average sea ice thickness from the GFDL model output. This research was funded by NSF ARCSS Grant DPP-9113851 and a grant from the NOAA Office of Global and Climate Change.

\section{APPENDIX}

\section{On the Timescale and Variance of Arctic Sea Ice Thickness}

A simple linear stochastic model for the response of the annual mean sea ice volume anomaly $h_{n}^{\prime}$ to atmospheric forcing can be written

$$
h_{n}^{\prime}=a h_{n-1}^{\prime}+Z_{n-1},
$$

where the ice volume is assumed to depend only on the ice volume in the previous year $h_{n-1}^{\prime}$ plus the annual integral of the atmospheric forcing felt by the sea ice, $Z$. If $Z$ has the characteristics of white noise, then Eq. (A1) describes a discrete first-order Markov process that has a red-noise power spectrum,

$$
H(f)=\frac{c}{1+a^{2}-2 a \cos (2 \pi f \Delta t)},
$$

where $c$ is a linear fitting constant related to the total variance, $f$ is frequency, and the time step $\Delta t$ is $1 \mathrm{yr}$ (see Jenkins and Watts 1968). The nonlinear fitting parameter $a$ can be related to a characteristic timescale of the variance by $\tau=-\Delta t / \ln (a)$, which is the response time for ice to adjust to a perturbation. Here, $\tau$ depends on the snow depth, the vertical resolution of heat conduction in the ice, and the equilibrium ice thickness (see Bitz et al. 1996).

The power spectra of the yearly sea ice volume anomaly are plotted with the best fit to Eq. (A2) (see Fig. 1c). The best-fit value of $\tau$ for $h^{\prime}$ from the PCCM forced by the observed $D$ is $15 \mathrm{yr}$; $\tau$ from the GFDL output is $4 \mathrm{yr}$. When the ice physics package of the PCCM is modified to be identical thermodynamically to that in the GFDL model, the ice response time is $3.5 \mathrm{yr}$, which compares well with the response time of $4.0 \mathrm{yr}$ in the GCM. For the simple stochastic model Eq. (A1), the standard deviation of the yearly $h^{\prime}\left(\sigma_{h^{\prime}}\right)$ is related to the standard deviation of the atmospheric forcing $\left(\sigma_{z}\right)$ on the sea ice by

$$
\sigma_{h^{\prime}}=\sigma_{z} \sqrt{\frac{\tau}{2}} .
$$

Hence, the variability in $h^{\prime}$ from the PCCM is expected to be almost two times greater than that from the GFDL model because of the differences in the response time. Since the variability of $h^{\prime}$ in the PCCM forced by the observed $D$ is actually about four times greater than that from the GFDL model, the difference in $\sigma_{z}$ between the PCCM and GFDL climate model must explain the other factor of two. For both models, $Z$ depends on the atmospheric energy transport $D$ and the upper surface sea ice physics (ie., albedo, snow depth). Although the forcing is indirectly computed by the PCCM and GFDL climate model, it cannot be represented by model variables in a simple way. However, based on the substantial difference between the observed and GFDL $D$ (see Fig. $3 a)$, it is reasonable to expect $\sigma_{z}$ in the PCCM to be much greater than that in the GFDL climate model.

\section{REFERENCES}

Barsugli, J., 1995: Idealized models of intrinsic midlatitude atmosphere-ocean interaction. Ph.D. thesis, University of Washington, 189 pp. [Available from UMI Catalog 9609579.]

Bitz, C. M., D. S. Battisti, R. E. Moritz, and J. A. Beesley, 1996: Low-frequency variability in the arctic atmosphere, sea ice, and upper-ocean system. J. Climate, 9, 394-408.

Boyle, J. E., 1993: Sensitivity of dynamical quantities to horizontal 
resolution for a climate simulation using the ECMWF (cycle 33) model. J. Climate, 6, 796-815.

Flato, G. M., 1995: Spatial and temporal variability of arctic ice thickness. Ann. Glaciol. 21, 323-29.

Häkkinen, S., 1993: An Arctic source for the great salinity anomaly: A simulation of the Arctic ice-ocean system for 1955-1975. J. Geophys. Res., 98, 16 397-16 410.

—, and G. L. Mellor, 1990: One hundred years of Arctic ice cover variations as simulated by a one-dimensional, ice-ocean model. J. Geophys. Res., 95, 15 959-15969.

Held, I. M., and P. J. Phillipps, 1993: Sensitivity of the eddy momentum flux to meridional resolution in atmospheric GCMs. $J$. Climate, 6, 499-507.

Houghton, J. T., G. J. Jenkins, and J. J. Ephraums, Eds., 1990: Climate Change: The IPCC Scientific Assessment. Cambridge, 365 pp.

Hunt, B. G., B. Gordon, and H. L. Davies, 1995: Impact of the Greehouse Effect on sea ice characteristics and snow accumulation in the polar regions. Int. J. Climatol., 15, 3-23.

Jenkins, G. M., and D. G. Watts, 1968: Spectral Analysis and Its Applications. Holden-Day, $524 \mathrm{pp}$.

Lau, N. C., S. G. H. Philander, and M. J. Nath, 1992: Simulation of El Niño/Southern Oscillation phenomenon with a low-resolution coupled general circulation model of the global ocean and atmosphere. J. Climate, 5, 284-307.

MacKay, R. M., and M. A. K. Khalil, 1991: Theory and development of a one dimensional time dependent radiative convective climate model. Chemosphere, 22, 383-417.

Manabe, S. J., and R. J. Stouffer, 1996: Low-frequency variability of surface air temperature in a 1000-year integration of a coupled atmosphere-ocean-land model. J. Climate, 9, 376-393.

, - - M. J. Spellman, and K. Bryan, 1991: Transient responses of a coupled ocean-atmosphere model to gradual changes of atmospheric $\mathrm{CO}_{2}$. Part I. Annual mean response. J. Climate, 4, 785-818.

— M. J. Spellman, and R. J. Stouffer, 1992: Transcient responses of a coupled ocean-atmosphere model to gradual changes of atmospheric $\mathrm{CO}_{2}$. II. Seasonal response. J. Climate, 5, 105-126.

McLaren, A. S., R. H. Bourke, J. E. Walsh, and R. L. Weaver, 1994: Variability in sea-ice thickness over the North Pole from 1958 to 1992. Polar Oceans and Their Role in Shaping the Global Environment, O. M. Johannessen, R. D. Muench, and J. E. Overland, Eds., Amer. Geophys. Union, 363-371.

Mitchell, J. F. B., T. C. Johns, J. M. Gregory, and S. F. B. Tett, 1994: Climate response to increasing levels of greenhouse gases and sulfate aerosols. Nature, 376, 501-504.

Moritz, R. E., J. E. Curry, A. S. Thorndike, and N. Untersteiner, Eds., 1993: A research program on the surface heat budget of the Arctic Ocean. OAII Science Management Office, $34 \mathrm{pp}$.

Nakamura, N., and A. H. Oort, 1988: Atmospheric heat budgets of the polar regions. J. Geophys. Res., 93, 9510-9524.

Overland, J. E., and P. Turet, 1994: Variability of the atmospheric energy flux across $70 \mathrm{~N}$ computed from the GFDL data set. Polar Oceans and Their Role in Shaping the Global Environment, O. M. Johannessen, R. D. Muench, and J. E. Overland, Eds., Amer. Geophys. Union, $540 \mathrm{pp}$.

Reif, F., 1965: Fundamentals of Statistical and Thermal Physics. McGraw-Hill, $651 \mathrm{pp}$.

Semtner, A. J., 1976: A model for the thermodynamic growth of sea ice in numerical investigations of climate. J. Phys. Oceanogr., 6, 379-389.

Thorndike, A. S., 1992: A toy model linking atmospheric thermal radiation and sea ice growth. J. Geophys. Res., 97, 9401-9410.

Walsh, J. E., W. D. Hibler, and B. Ross, 1985: Numerical simultion of Northern Hemisphere sea ice variability 1951-1980. J. Geophys. Res., 90, 4847-4865. 